\title{
SLC2A2 (GLUT2) as a novel prognostic factor for hepatocellular carcinoma
}

\author{
Yun Hak Kim ${ }^{1, *}$, Dae Cheon Jeong ${ }^{2, *}$, Kyoungjune Pak ${ }^{3}$, Myoung-Eun Han ${ }^{1}$, Ji-Young \\ $\mathrm{Kim}^{1}$, Liu Liangwen', Hyun Jin Kim", Tae Woo Kim², Tae Hwa Kim6, Dong Woo Hyun ${ }^{7}$ \\ and Sae-Ock Oh ${ }^{1}$ \\ ${ }^{1}$ Department of Anatomy, School of medicine, Pusan National University, Yangsan, Republic of Korea \\ ${ }^{2}$ Department of Statistics, Korea University, Seoul 02841, Republic of Korea \\ ${ }^{3}$ Department of Nuclear Medicine, Pusan National University Hospital, Busan, Republic of Korea \\ ${ }^{4}$ Eco-friendly new materials research center, Korea Research Institute of Chemical Technology (KRICT), Daejeon, Republic of Korea \\ ${ }^{5}$ Department of Orthopaedic Surgery, Pusan National University Hospital, Yangsan, Republic of Korea \\ ${ }^{6}$ Department of Internal Medicine, Pusan National University Hospital, Busan, Republic of Korea \\ ${ }^{7}$ Department of General Surgery, Pusan National University Hospital, Busan, Republic of Korea \\ *These authors contributed equally to this work \\ Correspondence to: Sae-Ock Oh, email: hedgehog@pusan.ac.kr \\ Keywords: hepatocellular carcinoma (HCC), solute carrier 2A (SLC2A), glucose transporter (GLUT), the cancer genome \\ atlas (TCGA), 2-18fluoro-deoxy-D-glucose ( $\left.{ }^{18} F D G\right)$
}

Received: June 10, $2017 \quad$ Accepted: July 25, $2017 \quad$ Published: August 14, 2017

Copyright: Kim et al. This is an open-access article distributed under the terms of the Creative Commons Attribution License 3.0 (CC BY 3.0 ), which permits unrestricted use, distribution, and reproduction in any medium, provided the original author and source are credited.

\section{ABSTRACT}

High rates of glucose transport via solute carrier (SLC2A, GLUT) family members are required to satisfy the high metabolic demands of cancer cells, and because of this characteristic of cancer cells $2-{ }^{18}$ fluoro-deoxy-D-glucose $\left({ }^{18}\right.$ FDG)-PET has become a powerful diagnostic tool. However, its sensitivity for hepatocellular carcinoma (HCC) is lower than for other malignancies, which suggests SLC2A family members are differentially expressed in HCC. In the present study, the expression patterns of SLC2A family members in tumor tissues and their associations with HCC progression were analyzed using data obtained from The Cancer Genome Atlas (TCGA). It was found that the expression of SLC2A2 (GLUT2) was higher in HCC than those of other members of the SLC2A family. The associations of the expression levels of SLC2A family members and previously known prognostic factors with clinical stages were examined using the $T$-test or the Mann-Whitney $U$ test, and interestingly, SLC2A2 expression was found to be associated with an advanced clinical stage $(p=0.0015)$. Furthermore, Kaplan-Meier analysis using the log-rank or the Gehan-BreslowWilcoxon test showed SLC2A2 expression was positively associated with overall survival $(p<0.001$, Gehan-Breslow-Wilcoxon test and $p=0.0145$ by multivariate Cox regression). The prognostic significance of SLC2A2 was similar in both early and late stages. However, it was more significant in HCC patients without alcohol consumption history and hepatitis C infection. Taken together, SLC2A2 was associated with clinical stages and independently associated with overall survival in patients with HCC. We suggest that SLC2A2 be considered a new prognostic factor for HCC. 


\section{INTRODUCTION}

Cancer cells acquire energy from different various sources, including glucose and fructose, to satisfy their high metabolic demands, and glucose and related hexoses are transported into cells via glucose transporter (GLUT) family (Solute carrier, SLC2A family) [1, 2]. To date, 14 members of the GLUT family have been identified, and their expressions are known to be tissue dependent [3-8]. Glucose is transported to cancer cells by SLC2A proteins, and 2- ${ }^{18}$ fluoro-deoxy-D-glucose $\left({ }^{18} \mathrm{FDG}\right)$-PET fundamentally relies on this process $[9-12] .{ }^{18} \mathrm{FDG}$ is primarily transported to cells by SLC2A1 and/or SLC2A3, so that their expressions are the most famous and studied prognostic factors among the SLC2A family members $[9,10,13-19]$. However, the sensitivity of ${ }^{18}$ FDG-PET for hepatocellular carcinoma (HCC) is lower than those of other cancers [20,21], which suggests SLC2A family members are differentially expressed in HCC.

HCC is the fifth most common cancer in men and the seventh most common in women [22, 23]. Many HCC patients have advanced disease at time of diagnosis, and this results in poor prognoses and high mortalities [22, 24]. Reported incidence rates of HCC are particularly high in East Asia, including South Korea, and in the United States the incidence HCC and the rate of HCC-associated mortality continue to increase $[22,23$, 25-27]. Despite curative and palliative treatment options, survival is poor due to late diagnoses and the ability of HCC to develop chemoresistance [28, 29]. Accordingly, new diagnostic and therapeutic targets are needed to improve survival. Over recent years, many molecular targets have been identified to determine prognosis, but their supposed merits are controversial $[29,30]$.

In the present study, we examined mRNA expression levels of SLC2A family members in HCC because HCC showed lower sensitivity for ${ }^{18}$ FDG-PET, and its prognostic significance using data from The Cancer Genome Atlas (TCGA) HCC cohort [24, 31-37].

\section{RESULTS}

\section{Patient characteristics}

From the TCGA hepatocellular carcinoma (HCC) data, clinical data and gene expression data were analyzed (Table 1). Mean age of the 372 patients was 59.47 years, and $67.5 \%$ were males. Mean overall survival months was 26.62 months. The racial composition of the cohort was Caucasian $49.7 \%$ and Asian 42.5\%. Regarding diagnoses, $97.3 \%$ had HCC, $1.9 \%$ hepatocholangiocarcinoma (mixed), and 0.8\% fibrolamellar carcinoma. Stages I, II, III and IV accounted for $46.2 \%, 23.4 \%, 22.8 \%$ and $1.6 \%$, respectively. About $75 \%$ of patients had several risk factors, and $\sim 25 \%$ had no primary risk factor.

\section{Patient selection}

The total number of HCC patients in the HCC cohort was 372. Patient IDs of RNA-seq data and clinical data were matched. For two-sample location test, the exclusions were as follows (Supplementary Figure 1); [1] patients with hepatocholangiocarcinoma or fibrolamellar carcinoma $(n=11)$, [2] patients with unknown stage $(n=22)$, [3] patients with NA or -infinite (-Inf) values for each target gene $(n=10)$. For multivariate regression analysis, NA and -Inf values of all target genes were excluded at once $(n=16)$.

\section{Expressions of SLC2A family members and their associations with clinical stages}

The mRNA expression levels of SLC2A family members in tumor tissues were analyzed using TCGA data (Figure 1A). Interestingly, the expression level of SLC2A2 (GLUT2) was higher than those of other members of the SLC2A family, and SLC2A14 was expressed the least (Figure 1A). To evaluate associations of the expression levels of SLC2A family members and previously known prognostic factors of $\mathrm{HCC}$ with clinical stages, we drew a box/scatter-plot (Figure 1B, 1C; Supplementary Figure 2, Figure 3) and conducted the two-sample location test (Figure 1B, 1C; Supplementary Tables 1, 2). If gene expressions were not normally distributed, we used the Mann-Whitney $U$ test. If gene expressions were normally distributed and had equal variance, $T$-test was used. $T$-test with Satterthwaite approximation was used in the case of normally distributed and no equal variance. As shown in Figure 1, the expressions of SLC2A1 was found to be positively correlated with advanced stage HCC ( $p=0.0079$, Mann-Whitney $U$ test $)$, however the expression of SLC2A2 was found to be negatively associated with advanced stage $(p=0.0015$, MannWhitney $U$ test). To assess racial differences of SLC2A2 expression, we analyzed the mean SLC2A2 expression values. The mean SLC2A2 expression levels of each races were similar except 'American indian or Alaska native', because there are only 2 patients (Supplementary Table 3).

\section{Association between SLC2A family members and survival}

Associations between gene expressions and patient survival were evaluated using Kaplan-Meier plots. Before the analysis, we divided the expression level of SLC2A1 or SLC2A2 into two (median expression group, Figure 2A, 2C) or four (quantile expression group, Figure 2B, 2D) groups. The Gehan-Breslow-Wilcoxon test was used for the most analysis, because survival curves were non-parallel (Figure 2B-2D and Tables 2, 3). However, the log-rank test was used for SLC2A1 
Table 1: Patient characteristics in the TCGA cohort

\begin{tabular}{|c|c|c|c|c|}
\hline & & & Total & $\%$ \\
\hline \multicolumn{3}{|c|}{ Age $($ mean $\pm \mathrm{SD}, n=372)$} & $59.47 \pm 13.49$ & - \\
\hline \multicolumn{3}{|c|}{ Overall survival_months (mean $\pm \mathrm{SD}, n=372$ ) } & $26.62 \pm 24.12$ & - \\
\hline \multirow{2}{*}{$\begin{array}{c}\text { Sex } \\
(n=372)\end{array}$} & \multicolumn{2}{|c|}{ Male } & 251 & 67.5 \\
\hline & \multicolumn{2}{|c|}{ Female } & 121 & 32.5 \\
\hline \multirow{5}{*}{$\begin{array}{l}\text { AJCC stage } \\
(n=372)\end{array}$} & \multicolumn{2}{|c|}{$\mathrm{I}$} & 172 & 46.2 \\
\hline & \multicolumn{2}{|c|}{ II } & 87 & 23.4 \\
\hline & \multicolumn{2}{|c|}{ III } & 85 & 22.8 \\
\hline & \multicolumn{2}{|c|}{ IV } & 6 & 1.6 \\
\hline & \multicolumn{2}{|c|}{ Unknown } & 22 & 5.9 \\
\hline \multirow{5}{*}{$\begin{array}{c}\text { Race } \\
(n=372)\end{array}$} & \multicolumn{2}{|c|}{ White } & 185 & 49.7 \\
\hline & \multicolumn{2}{|c|}{ Asian } & 158 & 42.5 \\
\hline & \multicolumn{2}{|c|}{ Black or American } & 17 & 4.6 \\
\hline & \multicolumn{2}{|c|}{ American Indian or Alaska native } & 2 & 0.5 \\
\hline & \multicolumn{2}{|c|}{ Unknown } & 10 & 2.7 \\
\hline \multirow{3}{*}{$\begin{array}{c}\text { Histological } \\
\text { Diagnosis } \\
(n=372)\end{array}$} & \multicolumn{2}{|c|}{ Hepatocellular carcinoma } & 362 & 97.3 \\
\hline & \multicolumn{2}{|c|}{ Hepatocholangiocarcinoma (mixed) } & 7 & 1.9 \\
\hline & \multicolumn{2}{|c|}{ Fibrolamellar carcinoma } & 3 & 0.8 \\
\hline \multirow{12}{*}{ Risk factors } & \multirow{5}{*}{ Alcohol consumption } & Alone & 68 & 58.1 \\
\hline & & Hepa B & 20 & 17.1 \\
\hline & & Hepa C & 14 & 12.0 \\
\hline & & Hepa B + C & 3 & 2.6 \\
\hline & & Others & 12 & 10.3 \\
\hline & \multirow{3}{*}{ Hepa B } & Alone & 76 & 93.8 \\
\hline & & Hepa C & 3 & 3.7 \\
\hline & & Others & 2 & 2.5 \\
\hline & \multirow{2}{*}{ Hepa C } & Alone & 32 & 97.0 \\
\hline & & Others & 1 & 3.0 \\
\hline & \multicolumn{2}{|c|}{ Others alone } & 30 & - \\
\hline & \multicolumn{2}{|c|}{ No primary risk factors } & 91 & - \\
\hline
\end{tabular}

(median expression group) because their survival curves were parallel (Figure 2A). Among previously known 31 prognostic genes examined, only 6 genes were found to be associated with survival and their results except SLC2A1 and SLC2A2 are shown in Supplementary Figures 4, 5 and Supplementary Tables 4, 5. In median subgroups, high expressions of SLC2A1 was associated with poor overall survival ( $p<0.001$, Figure 2A), whereas low SLC2A2 expression was associated with poor overall survival ( $p<$ 0.001 , Figure $2 \mathrm{C}$ ). In case of quantile subgroups, SLC2A2 expression level was associated with overall survival between each quantile groups, but SLC2A1 was not (Figure 2B, 2D and Table 2).

Furthermore, median patient survival was different depending on the expression levels of genes (Table 2;
Supplementary Tables 4,5 ). Notably, the difference in median survival between the low and high expression groups was the widest in the SLC2A2 group. Median survival in the SLC2A2-high group was 83.18 months, whereas that in the SLC2A2-low group was 40.37 months. Median survival in the SLC2A1-high group was 51.25 months, and that in the SLC2A1-low group was 83.18 months. In addition, each quantile survival of SLC2A2 tended to be increased depending on gene expression levels (1st: 2nd : 3rd : 4th $=29.53: 45.89: 80.68: 83.18$ ), whereas quantile survival of SLC2A1 did not show the pattern (Figure 2B, 2D).

To further compare the prognostic accuracy as continuous value, we examined the $\mathrm{C}$-index in timedependent Area Under the Curve (AUC) and AUC values 
at 5 years for SLC2A family members or previously known prognostic genes (Figure 2E, 2F and Supplementary Tables 5, 6). Even though SLC2A1 showed a higher C-index value (0.657) than SLC2A2 (0.626), SLC2A2 has the highest AUC value $(0.658)$ at 5 years than other genes (Figure 2E and Supplementary Tables 6, 7).

In order to identify whether the prognostic significance of SLC2A2 on survival can change depending on stages, we divided patients into two groups (Stage I \& II vs Stage III \& IV) and then drew Kaplan-Meier survival curve (Figure 3). The prognostic significance of SLC2A2 was similar in both early and late stages.

There are three representative risk factors in $\mathrm{HCC}$ which are alcohol consumption, hepatitis $\mathrm{B}$ and $\mathrm{C}$ infection. We examined whether the presence or absence of risk factors can affect the prognostic significance of SLC2A2 expression. Interestingly, alcohol consumption history affected the prognostic significance of SLC2A2 (Figure 4A, 4B). Low SLC2A2 expression was correlated with poor overall survival in patients who had alcohol consumption history (Figure 4A, 4B and Table 3). Hepatitis $\mathrm{B}$ infection did not affect the prognostic significance of SLC2A2 (Figure 4C, 4D and Table 3). However, the presence or absence of hepatitis $\mathrm{C}$ infection affected the significance of SLC2A2. As shown in Figure 4F, in patient group who did not have hepatitis virus $\mathrm{C}$ infections, high SLC2A2 expression had good survival outcomes. Median survival of each group is presented in Table 3.

To assess prognostic significance of SLC2A2 depending on surgery type, we classified patients into segmentectomy or lobectomy group. Irrespective of surgery type, high SLC2A2 expression had good survival outcome in both groups $(p=0.07,0.005$ respectively, Supplementary Figure 6).

Since SLC2A1 and SLC2A2 are associated with survival of patients in the present study, we examined whether the combination of SLC2A2 and SLC2A1 has better prognostic significance than SLC2A2 alone. Patients were classified into 4 groups; 1) SLC2A1 $<$ median and SLC2A2 $<$ median, 2) SLC2A1 $\geq$ median and SLC2A2 < median, 3) SLC2A1 < median and SLC2A2 $\geq$ median, 4) SLC2A $1 \geq$ median and SLC2A2 $\geq$ median. As shown in Supplementary Figure 7, group 1 curve crossed group 2 curve from 30 to 60 months, which means the combination of SLC2A1 and SLC2A2 might not be able to discriminate prognosis of HCC patients. However, SLC2A2 alone discriminated prognosis of patients during all the period (Figure 2D).

\section{Multivariate analysis}

Multivariate regression analysis was conducted to confirm these associations with survival. We compared SLC2A1 or SLC2A2 with other clinicopathologic variables at a time. The analysis showed that SLC2A2 expression was an independent prognostic factor for survival outcome, with a hazard ratio of 0.9097 (0.8433-0.9814, $p=0.0145$ ) along with age and stage (Table 4).

\section{DISCUSSION}

Several staging (AJCC, Okuda, BCLC) and scoring (Milan, ALBI, Child-Pugh) systems are used for hepatocellular carcinoma (HCC), but somewhat
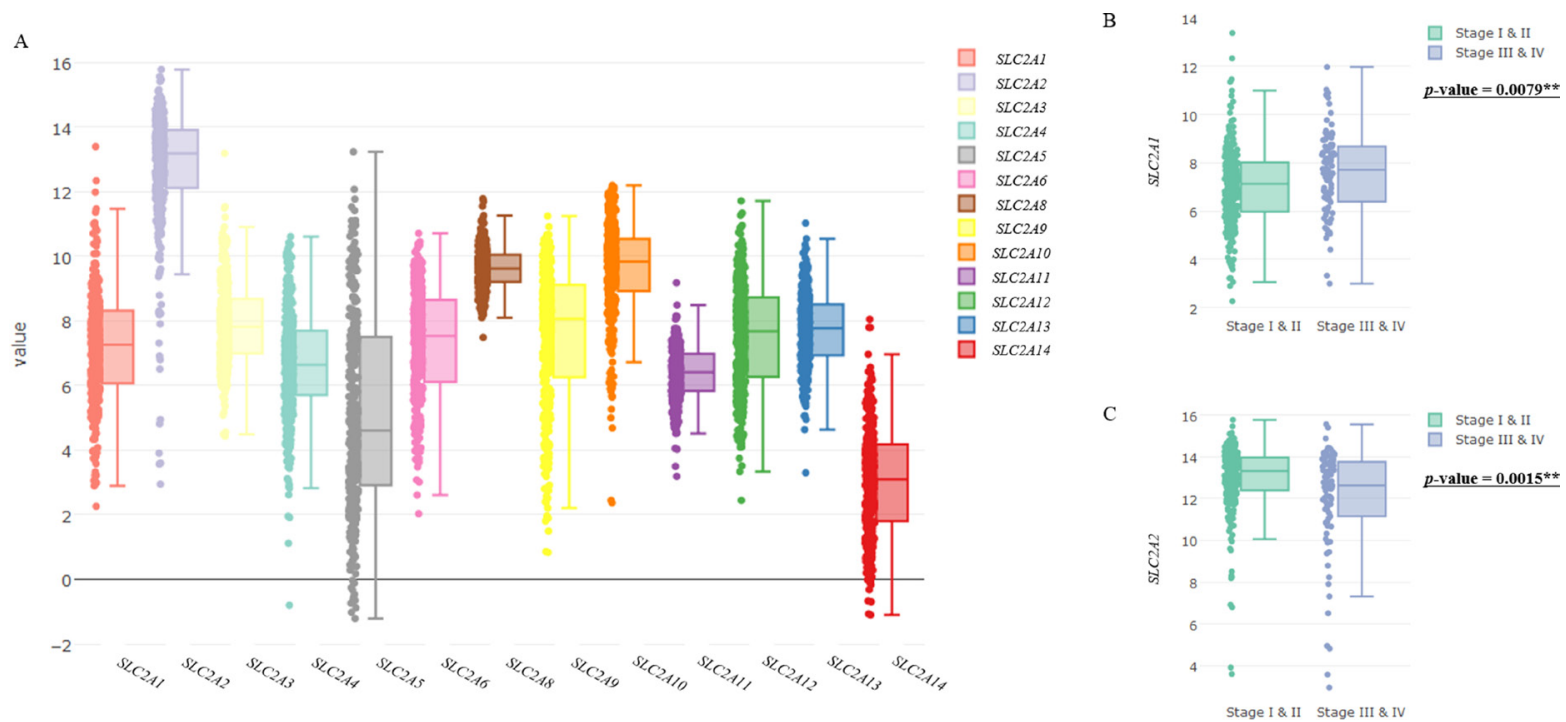

Figure 1: Expression levels of SLC2A family members in HCC and their association with clinical stages. (A) Boxplots represent mRNA expressions of SLC2A family members. (B, C) Relations between clinical stages and the expression levels of SLC2A1 (B) and SLC2A2 (C) were exhibited using boxplots and scatterplots. Central lines in boxes represent medians, boxes show interquartile ranges (IQR), and error bars show the full range of values, excluding outliers defined as being more than $\pm 1.5 \mathrm{IQR}$ outside boxes. Scatter plots represent raw data. The $p$-value in Figure 1B and 1C means results of two-sample location test of gene expression levels in Stage 1 \& II vs Stage III \& IV. 
A

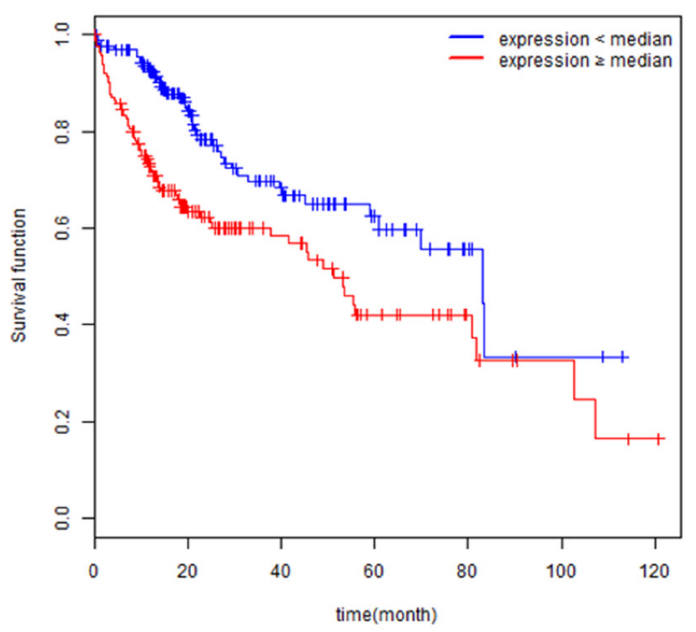

$\mathrm{C}$

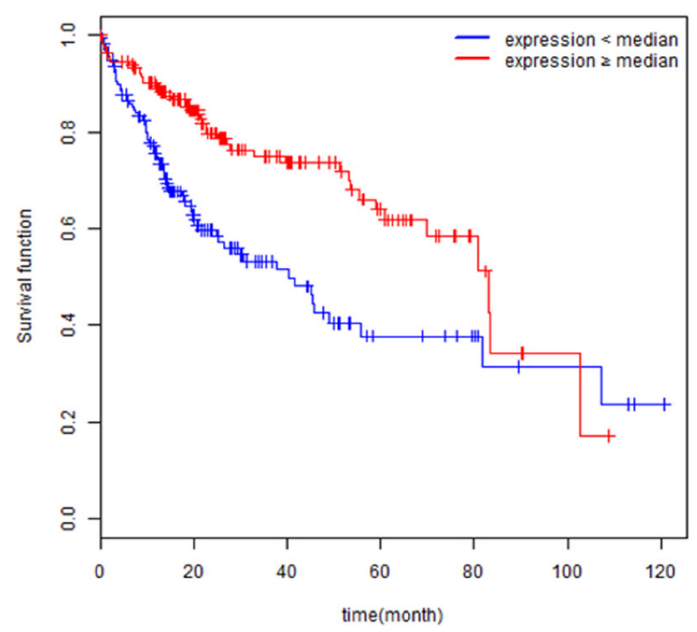

E

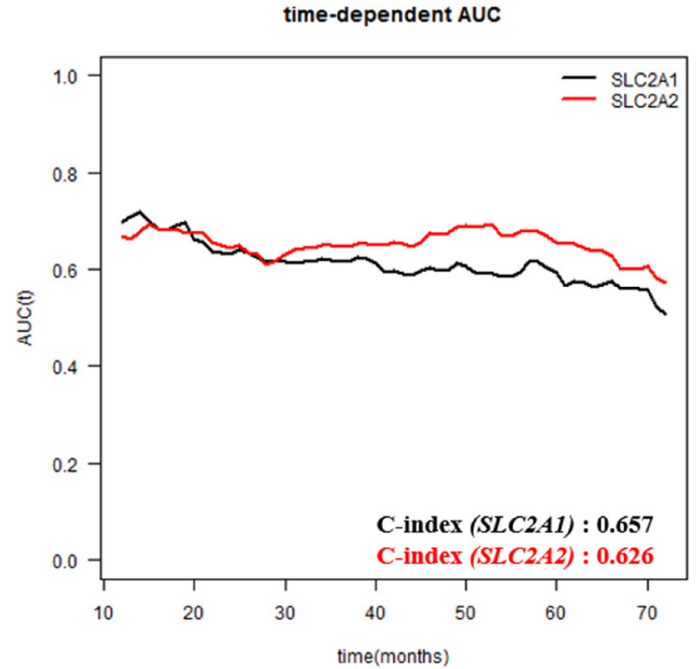

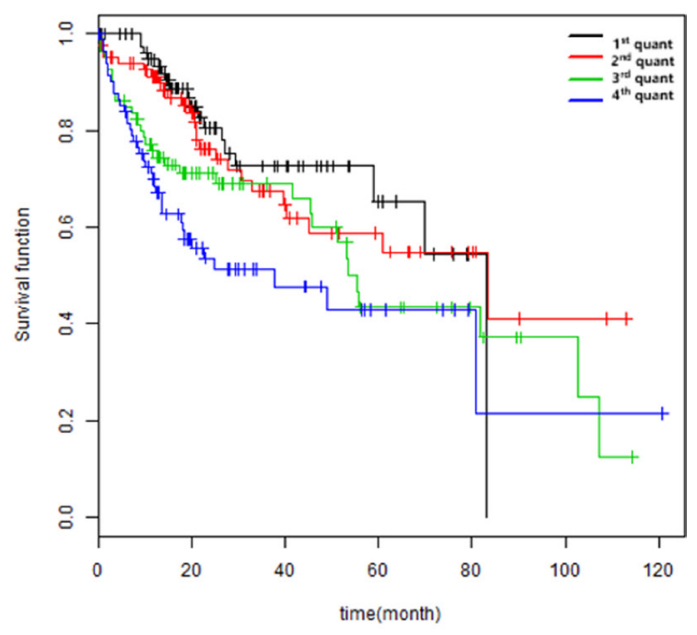

$\mathrm{D}$

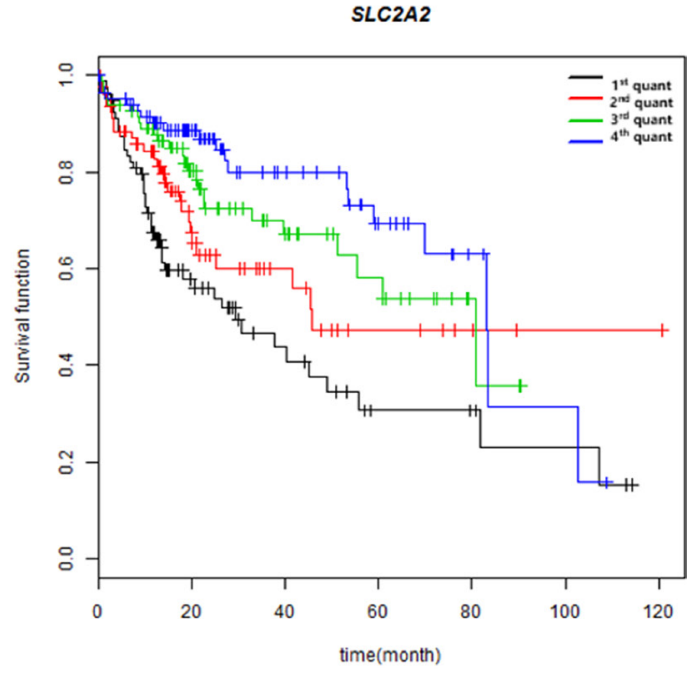

$\mathrm{F}$

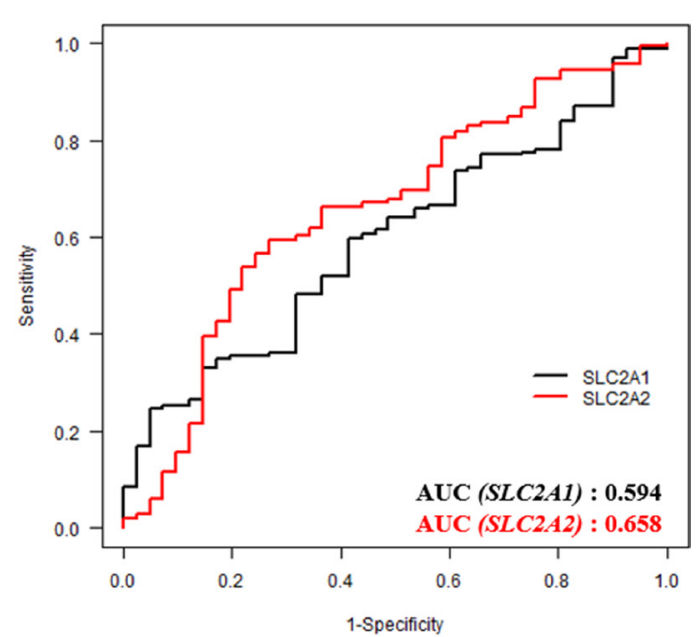

Figure 2: Survival analysis according to the expression levels of prognostic genes in patients with HCC. (A, B, C, D) Overall survival analysis of HCC patients with respect to the expression levels of SLC2A1 or SLC2A2 was performed by Kaplan-Meier analysis. (A, C) Expression levels of genes are classified into low or high compared with the median (blue or red lines, respectively). (B, D) Expression levels of genes are classified into four from lowest quantile (1st quant) to highest quantile (4th quant). (E, F) Time-dependent Area Under the Curve (AUC) and Receiver Operating Characteristic (ROC) curve at 5 years according to the continuous expression values of SLC2A1 or SLC2A2. Both C-index and AUC value at 5 years are described at the bottom right position of $\mathrm{E}$ and $\mathrm{F}$. 
Table 2: Test for equality of survival distributions for different levels of gene expression

\begin{tabular}{|c|c|c|c|c|c|c|c|}
\hline \multirow{2}{*}{$\begin{array}{l}\text { Gene } \\
\text { name }\end{array}$} & \multirow{2}{*}{$\begin{array}{c}\text { Protein } \\
\text { name }\end{array}$} & \multirow{2}{*}{$p$-value } & \multirow{2}{*}{ Test } & \multicolumn{4}{|c|}{ Median survival (months) } \\
\hline & & & & \multicolumn{2}{|c|}{ Low expression } & \multicolumn{2}{|c|}{ High expression } \\
\hline$S L C 2 A 1$ & GLUT1 & $<0.001 * * *$ & Log-rank & \multicolumn{2}{|c|}{83.18} & \multicolumn{2}{|c|}{51.25} \\
\hline$S L C 2 A 2$ & GLUT2 & $<0.001 * * *$ & Gehan-breslow-Wilcoxon & \multicolumn{2}{|c|}{40.37} & \multicolumn{2}{|c|}{83.18} \\
\hline \multirow{2}{*}{$\begin{array}{l}\text { Gene } \\
\text { name }\end{array}$} & \multirow{2}{*}{$\begin{array}{l}\text { Protein } \\
\text { name }\end{array}$} & \multirow{2}{*}{$p$-value } & \multirow{2}{*}{ Test } & \multicolumn{4}{|c|}{ Median survival (months) } \\
\hline & & & & 1st quant & 2nd quant & 3rd quant & 4th quant \\
\hline$S L C 2 A 1$ & GLUT1 & $<0.001 * * *$ & Gehan-breslow-Wilcoxon & 83.18 & 83.51 & 55.35 & 24.87 \\
\hline$S L C 2 A 2$ & GLUT2 & $<0.001 * * *$ & Gehan-breslow-Wilcoxon & 29.53 & 45.89 & 80.68 & 83.18 \\
\hline
\end{tabular}

Table 3: Test for equality of survival distributions for different levels of gene expression in each risk factor group and different stage group

\begin{tabular}{|c|c|c|c|c|c|c|}
\hline \multirow{2}{*}{$\begin{array}{l}\text { Gene } \\
\text { name }\end{array}$} & \multirow{2}{*}{$\begin{array}{c}\text { Protein } \\
\text { name }\end{array}$} & \multirow[b]{2}{*}{$p$-value } & \multirow[b]{2}{*}{ stage } & \multirow[b]{2}{*}{ Test } & \multicolumn{2}{|c|}{ Median survival } \\
\hline & & & & & $\begin{array}{c}\text { Low } \\
\text { expression }\end{array}$ & $\begin{array}{c}\text { High } \\
\text { expression }\end{array}$ \\
\hline$S L C 2 A 1$ & GLUT1 & $0.0062 * *$ & I \& II & Log rank & 83.18 & 55.68 \\
\hline$S L C 2 A 2$ & GLUT2 & $0.0050 * *$ & I \& II & Gehan-breslow-Wilcoxon & 45.89 & 83.18 \\
\hline$S L C 2 A 1$ & GLUT1 & $0.0264 *$ & III \& IV & Gehan-breslow-Wilcoxon & 30.58 & 18.27 \\
\hline$S L C 2 A 2$ & GLUT2 & $0.0025 * *$ & III \& IV & Gehan-breslow-Wilcoxon & 17.97 & 39.75 \\
\hline \multirow[b]{2}{*}{$\begin{array}{l}\text { Gene } \\
\text { name }\end{array}$} & \multirow[b]{2}{*}{$\begin{array}{c}\text { Protein } \\
\text { name }\end{array}$} & \multirow[b]{2}{*}{ Risk factor } & \multirow[b]{2}{*}{$p$-value } & \multirow[b]{2}{*}{ Test } & \multicolumn{2}{|c|}{ Median survival (months) } \\
\hline & & & & & $\begin{array}{c}\text { Low } \\
\text { expression }\end{array}$ & $\begin{array}{c}\text { High } \\
\text { expression }\end{array}$ \\
\hline \multirow{6}{*}{$S L C 2 A 2$} & \multirow{6}{*}{ GLUT2 } & Alcohol consumption (O) & 0.1675 & Gehan-breslow-Wilcoxon & 30.58 & 102.66 \\
\hline & & Alcohol consumption $(\mathrm{X})$ & $<0.001 * * *$ & Gehan-breslow-Wilcoxon & 41.75 & 80.68 \\
\hline & & Hepatitis B $(\mathrm{O})$ & $0.0131^{*}$ & Log rank & NA & NA \\
\hline & & Hepatitis B (X) & $0.0083 * *$ & Gehan-breslow-Wilcoxon & 30.58 & 70.01 \\
\hline & & Hepatitis C (O) & 0.1483 & Gehan-breslow-Wilcoxon & 25.23 & 60.84 \\
\hline & & Hepatitis C (X) & $<0.001 * * *$ & Gehan-breslow-Wilcoxon & 45.07 & 83.18 \\
\hline
\end{tabular}

surprisingly no consensus has been reached as which best predicts survival [38-40]. Because of limitations of conventional staging systems, new molecular markers need to be identified that can be used in combination with current staging systems. The present study suggests SLC2A2 (GLUT2) has potential as a novel prognostic factor in HCC and it could be applied for the imaging of HCC.

Most cancer cells exhibit high glucose metabolism. To obtain enough energy, cancer cells up-regulate the expressions of glucose transporters (GLUTs), especially SLC2A1, which has high affinity for glucose $[11,12,41]$.
SLC2A2 has relatively low affinity for glucose, mannose, galactose, and fructose, but high affinity for glucosamine $[8,42,43]$. As shown in Supplementary Table 8, different glucose transporters have different binding affinity for glucose $[6,7,44] .2-{ }^{18}$ Fluoro-deoxy-D-glucose $\left({ }^{18} \mathrm{FDG}\right)-$ PET-CT provides information about the metabolic statuses of tumors. FDG is primarily transported by SLC2A1 and SLC2A3 (Supplementary Table 8) [9, 10], which suggests that FDG uptake by tumor cells will be different depending on different expression levels of glucose transporters. Notably, the sensitivity of ${ }^{18}$ FDG-PET for 
HCC is lower than for other malignant cancers [20, 21], which suggests ${ }^{18}$ FDG (-) HCC does not overexpress SLC2A1 and SLC2A3. In the present study, we found the expression level of SLC2A2 was higher than those of SLC2A1 or SLC2A3 in HCC (Figure 1A).

Prediction of patient's survival is very important to make decisions about therapeutic methods. According to the predicted prognosis, clinicians determined the therapeutic options among curative (resection, transplantation and ablation), palliative (transarterial chemoembolization, sorafenib), and symptomatic treatments $[28,45]$. However, because there is no consensus on the use of prognostic prediction system, it is very important to develop prognostic markers. 5-year survival rate commonly used for estimating prognostic markers, because cancer-specific death usually occurs within 5 years, $25 \sim 50 \%$ in case of

A

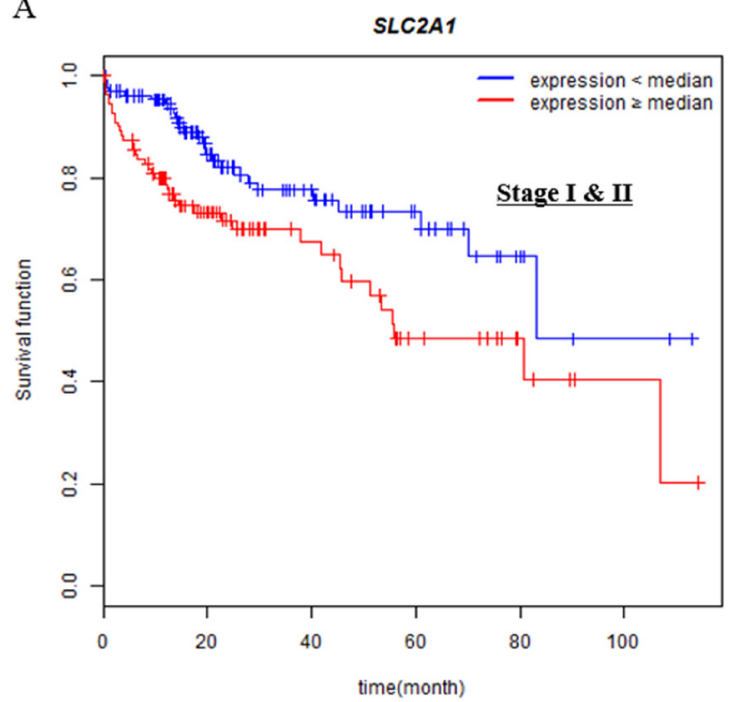

C

SLC2A2

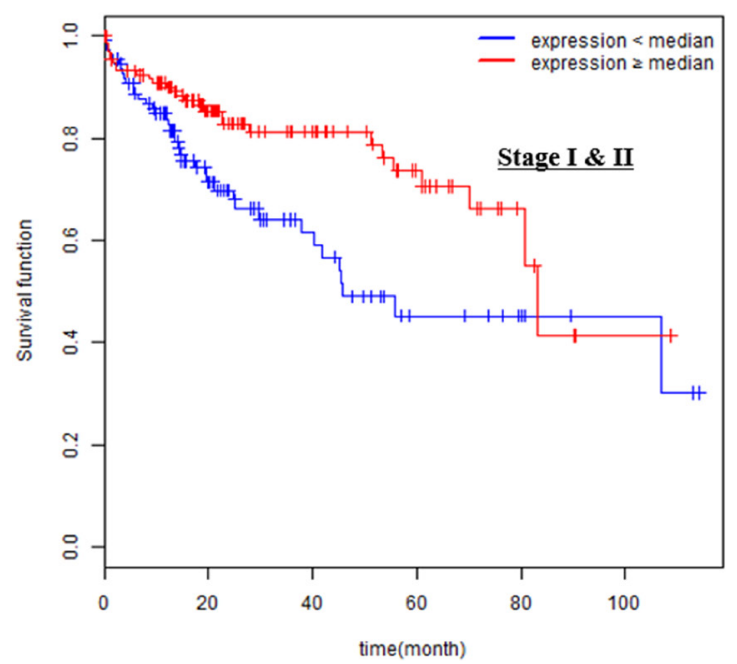

total HCC $[46,47]$. In the present study, we identified SLC2A2 has higher Area Under the Curve (AUC) value than other prognostic genes which suggests it may be very useful to predict 5-year survival rate of HCC.

Lower expression of SLC2A2 in $\mathrm{HCC}$ than in normal tissues suggests it could be used as an imaging target for diagnostic purposes [48, 49]. ${ }^{18}$ FDG-PET imaging is useful when cancers express high levels of SLC2A1 or SLC2A3. A specific imaging molecule targeting SLC2A2 could be used to detect $\mathrm{HCC}$ in a negative manner because SLC2A2 has relatively low affinity for glucose, mannose, galactose, and fructose. Furthermore, an imaging technique based on SLC2A2 could also be useful in renal cell carcinoma because SLC2A2 is mainly expressed in liver, absorptive renal cells, and pancreatic $\beta$ cells [50-52].

B

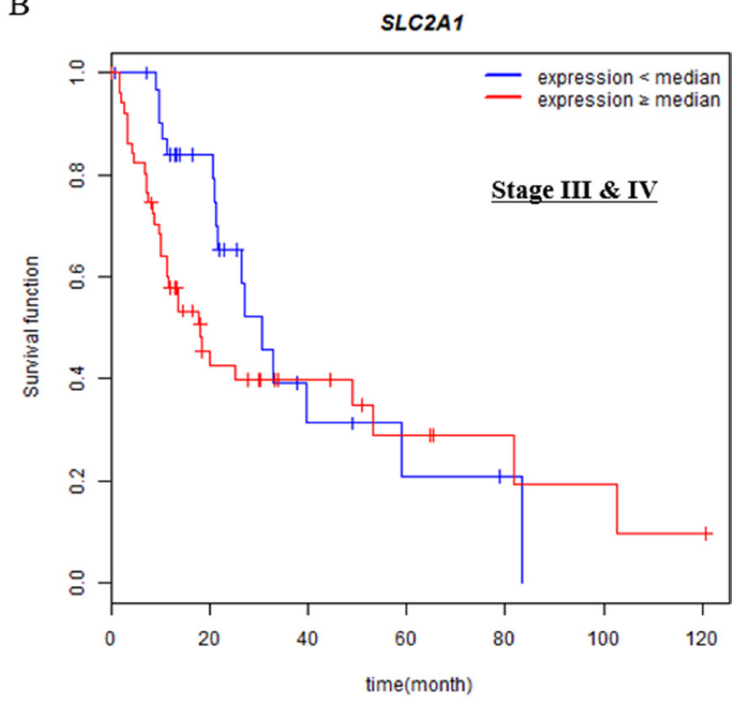

D

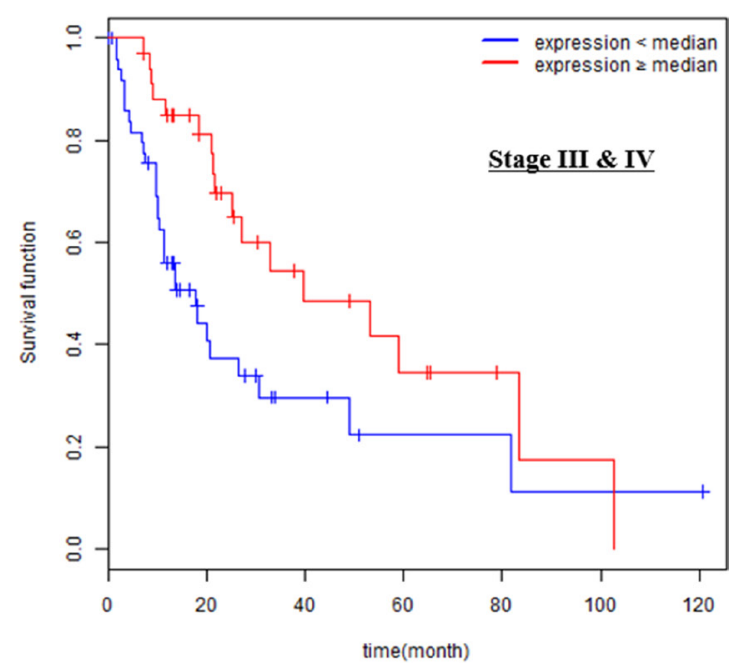

Figure 3: Associations of SLC2A1 or SLC2A2 expressions with overall survival in different tumor stages (I \& II vs III \& IV). Overall survival analysis of HCC patients with respect to the expression levels of SLC2A1 or SLC2A2 was performed by KaplanMeier analysis. Expression levels are classified into low or high compared with the median (blue or red lines, respectively). (A, C) The curves were analyzed in Stage I \& II group. (B, D) The curves were analyzed in Stage III \& IV group. 
Alcohol consumption and chronic viral hepatitis $\mathrm{B}, \mathrm{C}$ infections are the major known risk factors of $\mathrm{HCC}$ [53-55]. In the present study, we found the prognostic value of SLC2A2 was more significant in patients who has no major risk factors (Figure 4). Although SLC2A2 is not associated with overall survival of patients with alcohol consumption or viral hepatitis $\mathrm{C}$ infection, the high SLC2A2 expressions seemed to be correlated with good prognosis (Figure 4). Further studies are necessary in order to conclude SLC2A2 as a prognostic marker independent of risk factors.

Altered expressions of SLC2A family members have been reported in many types of cancer (liver- SLC2A1, SLC2A2, SLC2A5; pancreas- SLC2A1; breast- SLC2A1, $\underline{\text { Risk factor }(\mathbf{O})}$

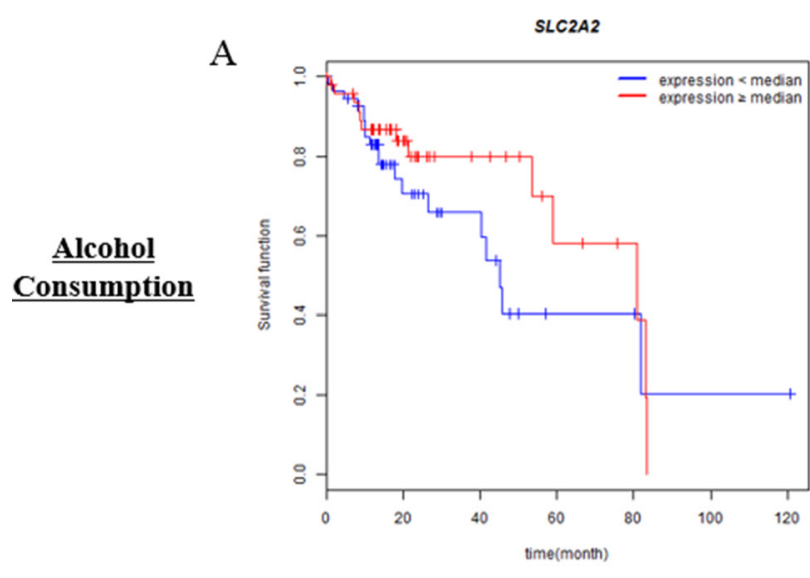

$\underline{\text { Hepatitis B }}$

$\underline{\text { Hepatitis C }}$

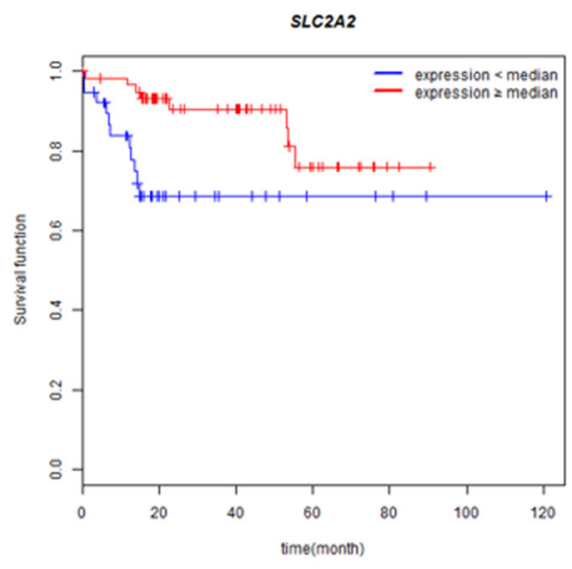

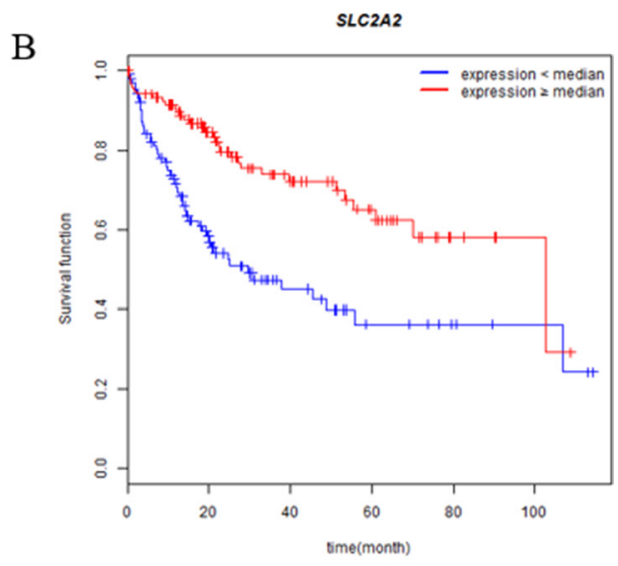
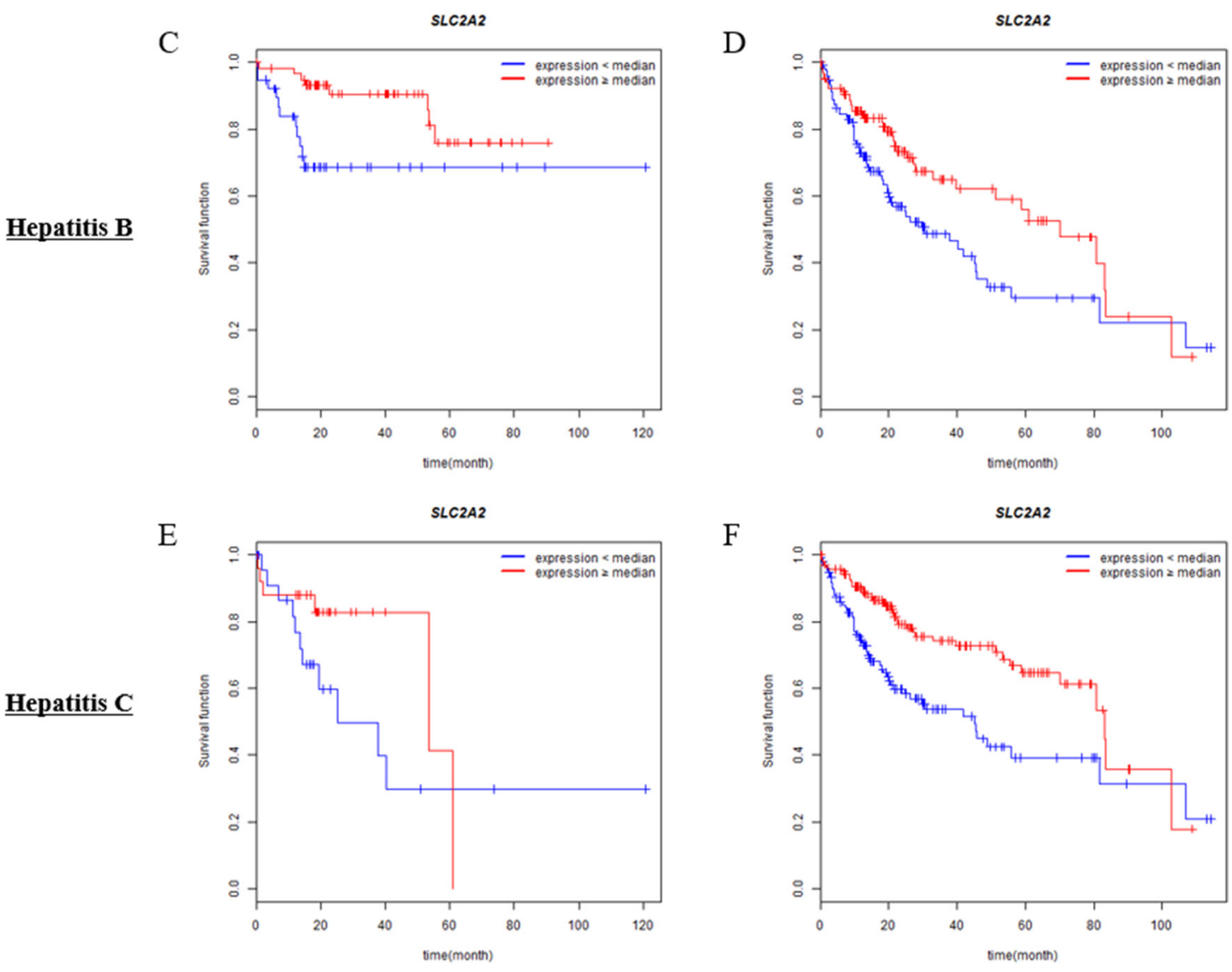

Figure 4: Associations of SLC2A1 or SLC2A2 expressions with overall survival in different risk factor groups. Overall survival analysis of HCC patients with respect to the expression levels of SLC2A1 or SLC2A2 was performed by Kaplan-Meier analysis. Expression levels are classified into low or high compared with the median (blue or red lines, respectively). Analysis was performed in groups in the presence $(\mathbf{A}, \mathbf{C}, \mathbf{E})$ or absence $(\mathbf{B}, \mathbf{D}, \mathbf{F})$ of risk factors (Alcohol consumption, Hepatitis B or $\mathrm{C}$ infection, respectively). 
Table 4: Multivariate analysis of relations between clinicopathological variables and overall survival

\section{Full model}

\begin{tabular}{cccccc}
\hline Clinicopathological variables & Total $\boldsymbol{N}$ & Hazard Ratio & $\boldsymbol{p}$-value & Lower 95\% & Upper 95\% \\
\hline SLC2A1 (GLUT1) & & 1.0043 & 0.9449 & 0.8881 & 1.1359 \\
SLC2A2 (GLUT2) & & 0.9096 & $0.0299^{*}$ & 0.8351 & 0.9908 \\
GENDER & \multirow{2}{*}{339} & 0.8409 & 0.1936 & 0.5745 & 1.2310 \\
AGE (continuous) & & 1.0096 & 0.1936 & 0.9952 & 1.0241 \\
STAGE (I or II vs III or IV) & & 2.3301 & $<0.001^{* * *}$ & 1.6015 & 3.3918 \\
\hline
\end{tabular}

Selected Model (using stepwise method)

\begin{tabular}{cccccc}
\hline Clinicopathological variables & Total $\boldsymbol{N}$ & Hazard Ratio & $\boldsymbol{p}$-value & Lower 95\% & Upper 95\% \\
\hline SLC2A2 (GLUT2) & & 0.9097 & $0.0145^{*}$ & 0.8433 & 0.9814 \\
AGE (continuous) & \multirow{2}{*}{339} & 1.0105 & 0.1459 & 0.9964 & 1.0249 \\
Stage (I or II vs III or IV) & & 2.3498 & $<0.001^{* * *}$ & 1.6154 & 3.4182 \\
\hline
\end{tabular}

SLC2A2, SLC2A4; stomach- SLC2A2, SLC2A4, SLC2A5, SLC2A14; lymphoma- SLC2A5). Interestingly, overexpression of some SLC2A isoforms have been shown to be of invasiveness and poor prognosis, especially SLC2A1 [7, 13-19, 56, 57]. However, expression of SLC2A2 was inversely associated with invasiveness in breast cancer [58]. Using the HCC dataset of TCGA, we compared SLC2A2 family and known prognostic factors for associations with survival. When we looked for gene expressional differences between stages I \& II vs stages III \& IV, interestingly, the $p$-value of SLC2A2 was more significant than those of any other putative prognostic factors (Figure 1, Supplementary Figures 1, 2 and Supplementary Tables 2, 3). Furthermore, the association between SLC2A2 and survival was supported by the survival analysis (Figure 2 and Table 2). As shown in Figure 2B, 2D (quantile survival curve), we identified overall survival of HCC patients was highly dependent on SLC2A2 expression compared to other prognostic genes.

The present study is the first to investigate the prognostic relevance of SLC2A2 in HCC, and we believe the results warrant further studies. We suggest that it could be a target for the diagnostic imaging of HCC in addition to its prognostic significance in $\mathrm{HCC}$.

\section{MATERIALS AND METHODS}

\section{Patients' data}

RNA-seq expression and clinical data for hepatocellular carcinoma (HCC) were downloaded from The Cancer Genome Atlas (TCGA, last download date: 2017.4.22) and cBio Cancer Genomics Portal (last download date: 2017.4.22). This process was performed by using ' $c g d s r$ ' package in R.

\section{Patient exclusion criteria}

Forty-nine patients in HCC cohort were excluded. The reasons for exclusion were: (1) not diagnosed as HCC, (2) staging not confirmed, and (3) Not available (NA) or - infinite (-Inf) gene expression values.

\section{Two-sample location test}

The only reason to choose one test over another in a given situation is its ability to reject a false hypothesis. The $T$-test is more powerful than the Mann-Whitney $U$ test when data are normally distributed, whereas $T$-test with Satterthwaite approximation (Welch's $T$-test) is more powerful when data are normally distributed and heterogeneous. On the other hand, the $T$-test is invalid when data are not normally distributed. For this reason, we first applied the Shapiro-Wilks normality test to gene expression and then conducted the two sample location test to gene expression between different stage groups. The boxplots were drawn by using 'plotly' package in R.

\section{Kaplan-Meier survival curves}

Median values are more robust against outliers than means. For this reason, medians can be used as measures when distributions are asymmetric or when one wishes to reduce the importance of outliers. The only reason to choose one test over another in given situations is if it will be more powerful, that is, more likely to reject a false hypothesis. The log-rank test is more powerful than Gehan's Wilcoxon test for detecting departures when two survival functions are parallel. On the other hand, Gehan's Wilcoxon test appears to be more powerful than the log-rank test for detecting other types of differences, for example, when survival functions are not parallel. 
Survival analysis was performed by using 'survMisc' and 'flexsurv' packages in R.

\section{Discriminatory accuracy analysis}

To evaluate the discriminatory accuracy as continuous value, we used UNO's C-index [59] in the time-dependent Area Under the Curve (AUC) analysis and AUC value in Receiver Operating Chracteristic (ROC) curve analysis at 5 years. These values were obtained using R package 'survival' and 'survAUC'.

\section{Multivariate Cox regression}

We used multivariate cox regression to compare the effect of SLC2A1, SLC2A2 on survival along with other clinicopathological factors (AJCC stage, age, gender).

\section{Abbreviations}

HCC; Hepatocellular carcinoma, SLC2A; Solute Carrier 2A; GLUT; Glucose transporter, TCGA; The Cancer Genome Atlas, SLC2A; Solute Carrier 2A, FDG; $2-{ }^{18}$ fluoro-deoxy-D-glucose

\section{Author contributions}

YHK: analyzed data and wrote manuscript; DCJ: collected and analyzed data; KP, MEH, JYK, LL: edited the manuscript and gave conceptual advice; HJK: gave technical support; TWK, THK, DWH: collected data and gave conceptual advice; SOO: supervised the project and wrote manuscript.

\section{CONFLICTS OF INTEREST}

The authors declare no competing financial interests.

\section{FUNDING}

This work was supported by the National Research Foundation of Korea (NRF-2016R1A2B4014593) and the Medical Research Center (MRC) Program through the National Research Foundation of Korea (NRF) grant funded by the Korea government (MSIP) (NRF2015R1A5A2009656). This research was supported by Basic Science Research Program through the National Research Foundation of Korea(NRF) funded by the Ministry of Education (NRF-2016R1A6A3A11931738).

\section{REFERENCES}

1. Avril N, Menzel M, Dose J, Schelling M, Weber W, Janicke F, Nathrath W, Schwaiger M. Glucose metabolism of breast cancer assessed by 18F-FDG PET: histologic and immunohistochemical tissue analysis. J Nucl Med. 2001; 42:9-16.

2. Zhu A, Lee D, Shim H. Metabolic positron emission tomography imaging in cancer detection and therapy response. Semin Oncol. 2011; 38:55-69.

3. Mueckler M, Thorens B. The SLC2 (GLUT) family of membrane transporters. Mol Aspects Med. 2013; 34:121-138.

4. Thorens B. GLUT2 in pancreatic and extra-pancreatic glucodetection (review). Mol Membr Biol. 2001; 18:265-273.

5. Thorens B, Mueckler M. Glucose transporters in the 21st Century. Am J Physiol Endocrinol Metab. 2010; 298:E141-145.

6. Joost H, Thorens B. The extended GLUT-family of sugar/ polyol transport facilitators: nomenclature, sequence characteristics, and potential function of its novel members (review). Mol Membr Biol. 2001; 18:247-256.

7. Pujol-Gimenez J, de Heredia FP, Idoate MA, Airley R, Lostao M, Evans AR. Could GLUT12 be a Potential Therapeutic Target in Cancer Treatment? A Preliminary Report. J Cancer. 2015; 6:139-143.

8. Uldry M, Ibberson M, Hosokawa M, Thorens B. GLUT2 is a high affinity glucosamine transporter. FEBS Lett. 2002; 524:199-203.

9. Avril N. GLUT1 expression in tissue and (18)F-FDG uptake. J Nucl Med. 2004; 45:930-932.

10. Khandani AH, Dunphy $\mathrm{CH}$, Meteesatien P, Dufault DL, Ivanovic M, Shea TC. Glut1 and Glut3 expression in lymphoma and their association with tumor intensity on 18F-fluorodeoxyglucose positron emission tomography. Nucl Med Commun. 2009; 30:594-601.

11. Pak K, Cheon GJ, Kang KW, Chung JK, Kim E, Lee DS. Prognostic value of SUVmean in oropharyngeal and hypopharyngeal cancers: comparison with SUVmax and other volumetric parameters of 18 F-FDG PET. Clin Nucl Med. 2015; 40:9-13.

12. Pak K, Cheon GJ, Nam HY, Kim SJ, Kang KW, Chung JK, Kim E, Lee DS. Prognostic value of metabolic tumor volume and total lesion glycolysis in head and neck cancer: a systematic review and meta-analysis. J Nucl Med. 2014; 55:884-890.

13. Higashi K, Ueda Y, Sakurai A, Wang XM, Xu L, Murakami M, Seki H, Oguchi M, Taki S, Nambu Y, Tonami H, Katsuda S, Yamamoto I. Correlation of Glut-1 glucose transporter expression with. Eur J Nucl Med. 2000; 27:1778-1785.

14. Koch A, Lang SA, Wild PJ, Gantner S, Mahli A, Spanier G, Berneburg M, Muller M, Bosserhoff A, Hellerbrand C. Glucose transporter isoform 1 expression enhances metastasis of malignant melanoma cells. Oncotarget. 2015; 6:32748-32760. https://doi.org/10.18632/oncotarget.4977.

15. Kunkel M, Reichert TE, Benz P, Lehr HA, Jeong JH, Wieand S, Bartenstein P, Wagner W, Whiteside TL. Overexpression of Glut-1 and increased glucose metabolism in tumors are associated with a poor prognosis in patients with oral squamous cell carcinoma. Cancer. 2003; 97:1015-1024. 
16. Macheda ML, Rogers S, Best JD. Molecular and cellular regulation of glucose transporter (GLUT) proteins in cancer. J Cell Physiol. 2005; 202:654-662.

17. Tian $\mathrm{M}$, Zhang $\mathrm{H}$, Nakasone $\mathrm{Y}$, Mogi $\mathrm{K}$, Endo $\mathrm{K}$. Expression of Glut-1 and Glut-3 in untreated oral squamous cell carcinoma compared with FDG accumulation in a PET study. Eur J Nucl Med Mol Imaging. 2004; 31:5-12.

18. Airley R, Loncaster J, Davidson S, Bromley M, Roberts S, Patterson A, Hunter R, Stratford I, West C. Glucose transporter glut-1 expression correlates with tumor hypoxia and predicts metastasis-free survival in advanced carcinoma of the cervix. Clin Cancer Res. 2001; 7:928-934.

19. Berlth F, Monig S, Pinther B, Grimminger P, Maus M, Schlosser H, Plum P, Warnecke-Eberz U, Harismendy O, Drebber U, Bollschweiler E, Holscher A, Alakus H. Both GLUT-1 and GLUT-14 are Independent Prognostic Factors in Gastric Adenocarcinoma. Ann Surg Oncol. 2015; 22:S822-831.

20. Khan MA, Combs CS, Brunt EM, Lowe VJ, Wolverson MK, Solomon H, Collins B, Di Bisceglie AM. Positron emission tomography scanning in the evaluation of hepatocellular carcinoma. J Hepatol. 2000; 32:792-797.

21. Kornberg A, Freesmeyer M, Barthel E, Jandt K, Katenkamp K, Steenbeck J, Sappler A, Habrecht O, Gottschild D, Settmacher U. 18F-FDG-uptake of hepatocellular carcinoma on PET predicts microvascular tumor invasion in liver transplant patients. Am J Transplant. 2009; 9:592-600.

22. El-Serag HB. Hepatocellular carcinoma. N Engl J Med. 2011; 365:1118-1127.

23. Venook AP, Papandreou C, Furuse J, de Guevara LL. The incidence and epidemiology of hepatocellular carcinoma: a global and regional perspective. Oncologist. 2010; 15:5-13.

24. Behne T, Copur MS. Biomarkers for hepatocellular carcinoma. Int J Hepatol. 2012; 2012:859076.

25. Altekruse SF, Henley SJ, Cucinelli J, McGlynn KA. Changing hepatocellular carcinoma incidence and liver cancer mortality rates in the United States. Am J Gastroenterol. 2014; 109:542-553.

26. Altekruse SF, McGlynn K, Reichman ME. Hepatocellular carcinoma incidence, mortality, and survival trends in the United States from 1975 to 2005. J Clin Oncol. 2009; 27:1485-1491.

27. Mittal S, El-Serag HB. Epidemiology of hepatocellular carcinoma: consider the population. J Clin Gastroenterol. 2013; 47:S2-6.

28. Bruix J, Sherman M, American Association for the Study of Liver D. Management of hepatocellular carcinoma: an update. Hepatology. 2011; 53:1020-1022.

29. Qin L, Tang ZY. The prognostic significance of clinical and pathological features in hepatocellular carcinoma. World J Gastroenterol. 2002; 8:193-199.
30. Llovet JM, Chen Y, Wurmbach E, Roayaie S, Fiel MI, Schwartz M, Thung SN, Khitrov G, Zhang W, Villanueva A, Battiston C, Mazzaferro V, Bruix J, et al. A molecular signature to discriminate dysplastic nodules from early hepatocellular carcinoma in $\mathrm{HCV}$ cirrhosis. Gastroenterology. 2006; 131:1758-1767.

31. Cancer Genome Atlas Research N, Weinstein JN, Collisson EA, Mills GB, Shaw KR, Ozenberger BA, Ellrott K, Shmulevich I, Sander C, Stuart JM. The Cancer Genome Atlas Pan-Cancer analysis project. Nat Genet. 2013; 45:1113-1120.

32. Cerami E, Gao J, Dogrusoz U, Gross BE, Sumer SO, Aksoy BA, Jacobsen A, Byrne CJ, Heuer ML, Larsson E, Antipin Y, Reva B, Goldberg AP, et al. The cBio cancer genomics portal: an open platform for exploring multidimensional cancer genomics data. Cancer Discov. 2012; 2:401-404.

33. Frau M, Biasi F, Feo F, Pascale RM. Prognostic markers and putative therapeutic targets for hepatocellular carcinoma. Mol Aspects Med. 2010; 31:179-193.

34. Gao J, Aksoy BA, Dogrusoz U, Dresdner G, Gross B, Sumer SO, Sun Y, Jacobsen A, Sinha R, Larsson E, Cerami E, Sander C, Schultz N. Integrative analysis of complex cancer genomics and clinical profiles using the cBioPortal. Sci Signal. 2013; 6:pl1.

35. Schmitz KJ, Sotiropoulos GC, Baba HA, Schmid KW, Muller D, Paul A, Auer T, Gamerith G, Loeffler-Ragg J. AKR1B10 expression is associated with less aggressive hepatocellular carcinoma: a clinicopathological study of 168 cases. Liver Int. 2011; 31:810-816.

36. Sun H, Teng M, Liu J, Jin D, Wu J, Yan D, Fan J, Qin X, Tang H, Peng Z. FOXM1 expression predicts the prognosis in hepatocellular carcinoma patients after orthotopic liver transplantation combined with the Milan criteria. Cancer Lett. 2011; 306:214-222.

37. Tsuchiya M, Parker JS, Kono H, Matsuda M, Fujii H, Rusyn I. Gene expression in nontumoral liver tissue and recurrence-free survival in hepatitis $\mathrm{C}$ virus-positive hepatocellular carcinoma. Mol Cancer. 2010; 9:74.

38. Liu PH, Hsu CY, Hsia CY, Lee YH, Su CW, Huang YH, Lee FY, Lin H, Huo TI. Prognosis of hepatocellular carcinoma: Assessment of eleven staging systems. J Hepatol. 2016; 64:601-608.

39. Marrero JA, Fontana RJ, Barrat A, Askari F, Conjeevaram HS, Su G, Lok AS. Prognosis of hepatocellular carcinoma: comparison of 7 staging systems in an American cohort. Hepatology. 2005; 41:707-716.

40. Sherman M. Staging for hepatocellular carcinoma: complex and confusing. Gastroenterology. 2014; 146:1599-1602.

41. Seo S, Hatano E, Higashi T, Nakajima A, Nakamoto Y, Tada M, Tamaki N, Iwaisako K, Kitamura K, Ikai I, Uemoto S. P-glycoprotein expression affects $18 \mathrm{~F}$-fluorodeoxyglucose accumulation in hepatocellular carcinoma in vivo and in vitro. Int J Oncol. 2009; 34:1303-1312. 
42. Mardones L, Ormazabal V, Romo X, Jana C, Binder P, Pena E, Vergara M, Zuniga FA. The glucose transporter-2 (GLUT2) is a low affinity dehydroascorbic acid transporter. Biochem Biophys Res Commun. 2011; 410:7-12.

43. Wu L, Fritz J, Powers AC. Different functional domains of GLUT2 glucose transporter are required for glucose affinity and substrate specificity. Endocrinology. 1998; 139:4205-4212.

44. Uldry M, Thorens B. The SLC2 family of facilitated hexose and polyol transporters. Pflugers Arch. 2004; 447:480-489.

45. Heimbach J, Kulik LM, Finn R, Sirlin CB, Abecassis M, Roberts LR, Zhu A, Murad M, Marrero J. Aasld guidelines for the treatment of hepatocellular carcinoma. Hepatology. 2017.

46. Lee JG, Kang CM, Park JS, Kim KS, Yoon DS, Choi JS, Lee W, Kim BR. The actual five-year survival rate of hepatocellular carcinoma patients after curative resection. Yonsei Med J. 2006; 47:105-112.

47. Nakakura E, Choti MA. Management of hepatocellular carcinoma. Oncology (Williston Park). 2000; 14:1085-1098; discussion 1098-1102.

48. Grobholz R, Hacker HJ, Thorens B, Bannasch P. Reduction in the expression of glucose transporter protein GLUT 2 in preneoplastic and neoplastic hepatic lesions and reexpression of GLUT 1 in late stages of hepatocarcinogenesis. Cancer Res. 1993; 53:4204-4211.

49. Kasai D, Adachi T, Deng L, Nagano-Fujii M, Sada K, Ikeda M, Kato N, Ide YH, Shoji I, Hotta H. HCV replication suppresses cellular glucose uptake through down-regulation of cell surface expression of glucose transporters. J Hepatol. $2009 ; 50: 883-894$.
50. Karim S, Adams D, Lalor PF. Hepatic expression and cellular distribution of the glucose transporter family. World J Gastroenterol. 2012; 18:6771-6781.

51. Leturque A, Brot-Laroche E, Le Gall M. GLUT2 mutations, translocation, and receptor function in diet sugar managing. Am J Physiol Endocrinol Metab. 2009; 296:E985-992.

52. Thorens B. GLUT2, glucose sensing and glucose homeostasis. Diabetologia. 2015; 58:221-232.

53. Fattovich G, Stroffolini T, Zagni I, Donato F. Hepatocellular carcinoma in cirrhosis: incidence and risk factors. Gastroenterology. 2004; 127:S35-50.

54. Gomaa AI, Khan SA, Toledano MB, Waked I, TaylorRobinson SD. Hepatocellular carcinoma: epidemiology, risk factors and pathogenesis. World J Gastroenterol. 2008; 14:4300-4308.

55. Williams R. Global challenges in liver disease. Hepatology. 2006; 44:521-526.

56. Medina R, Owen GI. Glucose transporters: expression, regulation and cancer. Biol Res. 2002; 35:9-26.

57. Szablewski L. Expression of glucose transporters in cancers. Biochim Biophys Acta. 2013; 1835:164-169.

58. Grover-McKay M, Walsh SA, Seftor EA, Thomas P, Hendrix MJ. Role for glucose transporter 1 protein in human breast cancer. Pathol Oncol Res. 1998; 4:115-120.

59. Uno H, Cai T, Pencina MJ, D’Agostino R, Wei LJ. On the C-statistics for evaluating overall adequacy of risk prediction procedures with censored survival data. Stat Med. 2011; 30:1105-1117. 